\title{
0corrência de Atrazina em águas no Brasil e remoção no tratamento da água: revisão sistemática
}

\author{
Agata Cristina Lima Dias ${ }^{1 ;}$ Juliana Mattos Bohrer Santos ${ }^{2}$; Ana Silvia Pereira Santos ${ }^{3}$; Sue Ellen \\ Costa Bottrel'; Renata de Oliveira Pereira ${ }^{l}$ \\ ana.pereira@uerj.br
}

1. Universidade Federal de Juiz de Fora, MG, Brasil $\quad$ 2. Universidade Federal de Minas Gerais, MG, Brasil

3. Universidade do Estado do Rio de Janeiro, RJ, Brasil

Histórico do Artigo:

Recebido em: 16 de maio de 2018 Aceito em: 18 de setembro de 2018 Publicado em: 28 de dezembro de 2018

Resumo: A atrazina é um dos agrotóxicos mais utilizados no Brasil e possui grande tendência a contaminar águas superficiais e subterrâneas. Logo, o objetivo do estudo foi realizar uma revisão bibliográfica para verificar a ocorrência da atrazina em águas superficiais, subterrâneas e tratadas no Brasil e sua remoção durante 0 tratamento de água. Para tanto, buscaram-se trabalhos científicos publicados entre os anos de 2000 e 2017 que trataram sobre a presença do composto nas matrizes supracitadas. Para a análise da remoção da atrazina em água buscaram-se trabalhos que empregaram processos como tratamento convencional, ozonização, fotólise, adsorção, nanofiltração, entre outros. Utilizaram-se como plataformas de pesquisa o Periódicos Capes, Google Acadêmico e Science Direct. Analisaram-se 35 trabalhos referentes a presença do composto em águas no Brasil e 43 sobre sua remoção da água. 0bservou-se nos trabalhos, frequências de detecção da atrazina de 8\% para águas superficiais e $12 \%$ para subterrâneas e ocorrência em valores superiores ao VMP em ambas as matrizes. Em águas tratadas a presença, bem como concentração da atrazina foi reportada em dois estudos, porém, em concentrações inferiores ao VMP. 0 tratamento de ciclo completo é ineficaz para a remoção da atrazina. Sendo assim, é necessária a associação de outros processos ao tratamento convencional, dentre os quais: a adsorção em carvão ativado, ozonização, nanofiltração, osmose inversa (OI) e processos oxidativos avançados (P0As). Porém, é importante se atentar à conversão dos contaminantes a subprodutos que podem apresentar toxicidade.

Palavras-chave: Agrotóxico, Tratamento Convencional, Subprodutos, Saúde.

\section{0ccourrence of Atrazine in brazilian water and removal by watertreatment: systematic review}

\begin{abstract}
Atrazine is one of the most commonly used agrochemicals in Brazil and has a high tendency to contaminate surface and groundwater. Therefore, the objective of the study was to perform a bibliographic review to verify the occurrence of atrazine in surface, groundwater and treated waters in Brazil and its removal during the water treatment. Therefore, scientific literature published between 2000 and 2017 that dealt with the presence of the compound in the above mentioned matrices were searched. For the removal analysis of atrazine in water, studies that used processes such as conventional treatment, ozonization, photolysis, adsorption, nanofiltration, among others, were searched. Capes, Google Scholar and Science Direct were used as research platforms. A total of 35 studies on the presence of the compound in water in Brazil were analyzed, and 43 on its removal from the water. Atrazine detection frequencies about $8 \%$ for surface water and $12 \%$ for groundwater were observed in the reviewed studies, in addition to their occurrence at values higher than MPV in both matrices. In treated waters the presence and concentration of atrazine was reported in two studies, but at concentrations below the MPV. Full-cycle treatment is ineffective for the removal of atrazine. Therefore, it is necessary to associate other processes to the conventional treatment, among which are cited: activated carbon adsorption, ozonation, nanofiltration, reverse osmosis (R0) and advanced oxidative processes (AOPs). However, it is important to pay attention to the conversion of the contaminants to by-products that can be toxic.
\end{abstract}

Keywords: Agrochemicals, Conventional Treatment, By-products, Health. 


\title{
0currencia del Atrazina en aguas en Brasil y remoción en el tratamiento del agua: revisión sistemática
}

\begin{abstract}
Resumen: La atrazina es uno de los agrotóxicos más utilizados en Brasil y tiene gran tendencia a contaminar aguas superficiales y subterráneas. El objetivo del estudio fue realizar una revisión bibliográfica para verificar la ocurrencia de la atrazina en aguas superficiales, subterráneas y tratadas en Brasil y su remoción durante tratamiento del agua. Para tanto, se buscaron trabajos científicos publicados entre los años de 2000 y 2017 que trataron sobre la presencia del compuesto en las matrices citadas. Para análisis de la remoción de la atrazina en agua se buscaron trabajos que emplearon procesos como tratamiento convencional, ozonización, fotolisis, adsorción, nanofiltración, entre otros. Se utilizaron como plataformas de investigación el Periódicos Capes, Google Académico y Science Direct. Se analizaron 35 trabajos referentes a la presencia del compuesto en aguas en Brasil y 43 sobre su remoción del agua. Se observó en los trabajos, frecuencias de detección de la atrazina de 8\% para aguas superficiales y $12 \%$ para subterráneas e su ocurrencia en valores superiores al VMP en ambas las matrices. En aguas tratadas la presencia, así como concentración de la atrazina fue reportada en dos estudios, pero en concentraciones inferiores alo VMP. El tratamiento de ciclo completo es ineficaz para la eliminación de la atrazina. Por eso, es necesaria la asociación de otros procesos al tratamiento convencional, entre los cuales: la adsorción en carbón activado, ozonización, nanofiltración, osmosis inversa (0I) y procesos oxidativos avanzados (P0As). Sin embargo, es importante atentar para la conversión de los contaminantes a subproductos que pueden tener toxicidad.
\end{abstract}

Palabras clave: Agrotóxico, Tratamiento Convencional;Subproductos, Salud.

\section{INTRODUÇÃ0}

0 uso de agrotóxicos no Brasil ocorre de forma acentuada, sendo o país o maior consumidor destes produtos no mundo (BRASIL, 2018a). No ano de 2016 a venda foi de aproximadamente 500 mil toneladas (BRASIL, 2018b). A necessidade de maior produção de alimentos faz com que a utilização de agrotóxicos seja a alternativa mais atrativa para os produtores. Contudo, sua ingestão através de alimentos ou água contaminados representa um potencial impacto para a saúde humana (BAIRD; CANN, 2011).

No Brasil, a Lei $\mathrm{n}^{\circ} 7.802$ de 11 de julho 1989, que trata sobre o uso de agrotóxicos, abrange desde as pesquisas, experimentações e produção até o destino das embalagens, controle e fiscalização de agrotóxicos no país (BRASIL, 1989). Sendo regulamentada pelo Decreto n ${ }^{\circ} 4.070$ de 4 de janeiro de 2002, a legislação determina ao Ministério da Agricultura, Pecuária e Abastecimento (MAPA), ao Ministério do Meio Ambiente (MMA) e ao Ministério da Saúde (MS), dentre outras responsabilidades, o estabelecimento do limite máximo de resíduos e 0 intervalo de concentração de segurança dos agrotóxicos e afins (BRASIL, 2002). Portanto, com este propósito, a Portaria de Consolidação (PCR) MS n ${ }^{0}$ 5, de 28 de setembro de 2017, Anexo XX estabelece os padrões de potabilidade da água no país, incluindo os valores máximos permitidos (VMP) para a concentração de 38 agrotóxicos em águas de abastecimento humano (BRASIL, 2017). 
Dentre os agrotóxicos abordados pela PRC MS n ${ }^{0} 5$ de 2017, Anexo XX, encontra-se a atrazina, um herbicida amplamente utilizado no país. Segundo dados de comercialização disponibilizados pelo Instituto Brasileiro de Meio Ambiente e dos Recursos Naturais (IBAMA) entre os anos de 2009 e 2016, o composto esteve na lista dos 10 princípios ativos (P.A.) mais vendidos no país. Nesse período foram vendidas aproximadamente 160 mil toneladas de P.A., sendo a atrazina o quarto mais vendido no ano de 2016 (BRASIL, 2018b).

A atrazina não apresenta potencial carcinogênico ao ser humano, embora apresente potencial impacto relacionado à reprodução e desenvolvimento humano (NHMRC/ NRMMC, 2011; JOWA; HOWD, 2011 apud MARIN-MORALES; VENTURA-CAMARG0; HOSHINA, 2013). Em uma revisão crítica da literatura Solomon et al. (2008) concluíram que o composto, em concentrações ambientalmente relevantes, não afeta o crescimento, desenvolvimento sexual, reprodução e sobrevivência de anfíbios, peixes e répteis aquáticos. Efeitos tóxicos da atrazina foram reportados em planta (Tradescantia clone 4430) e molusco asiático Corbicula fulminea (Patussi e Bündchen, 2013; dos Santos e Martinez, 2014).

0 composto é classificado pela ANVISA como classe III de toxicidade, na qual se enquadram os compostos medianamente tóxicos (BRASIL, 2018c). Segundo Carmo et al. (2013) a atrazina pode possuir toxidade crônica sobretudo nos sistemas hormonal e reprodutor. A ingestão do composto em concentrações acima do VMP por muitos anos pode acarretar problemas cardiovasculares e dificuldades reprodutivas (USEPA, 2018a). A exposição ao agrotóxico pode ter efeitos como irritação da pele, falta de ar, espasmos musculares e problemas genéticos (USEPA, 2018b).

No Brasil o VMP para o composto em água potável é de $2 \mu \mathrm{g} / \mathrm{L}($ BRASIL, 2017). A Organização Mundial da Saúde (0MS) (WH0, 2017) determina o valor de $100 \mu \mathrm{g} / \mathrm{L}$, correspondente à soma de atrazina e seus metabólitos cloro-s-triazinas. Este também é critério adotado pela Nova Zelândia (MINISTRY OF HEALTH, 2017). As normas do Canadá, Austrália e Estados Unidos estabelecem, respectivamente, os seguintes VMP: $5 \mu \mathrm{g} / \mathrm{L}$ (atrazina e metabolitos), $20 \mu \mathrm{g} / \mathrm{L}$ e $3 \mu \mathrm{g} / \mathrm{L}$ (HEALTH CANADA, 2017; NHMRC/NRMMC, 2011; USEPA, 2018b). Já a União Européia define valores mais restritivos, onde a soma da concentração de agrotóxicos em água potável não deve ultrapassar $0,5 \mu \mathrm{g} / \mathrm{L}$, sendo que as concentrações individuais devem ser inferiores a 0,1 $\mu \mathrm{g} / \mathrm{L}$ (EUROPEAN UNION, 1998).

Devido à ampla utilização da atrazina no país e possíveis efeitos adversos à saúde, que sua ingestão pode acarretar, é importante realizar uma avaliação do risco de contaminação da água destinada ao consumo humano. A metodologia de Avaliação de Risco pressupõe quatro 
0corrência de Atrazina em águas no Brasil e remoção no tratamento da água: revisão sistemática

etapas fundamentais (i) identificação do perigo, (ii) avaliação da dose-resposta, (iii) avaliação da exposição e (iv) caracterização do risco (WH0, 2017). Diante do exposto, esse artigo visa realizar a avaliação da exposição à atrazina através de dados sobre sua dinâmica ambiental, sua ocorrência em corpos d'água brasileiros e da possibilidade de sua remoção pelo tratamento da água. Ainda, o trabalho tem o objetivo de verificar a formação de subprodutos da atrazina nos processos de tratamento de água.

\section{MATERIAL E MÉTODOS}

A dinâmica ambiental da atrazina foi analisada segundo os seguintes parâmetros:

- $\quad$ KOC- coeficiente de adsorção ao carbono orgânico do solo;

- $\quad$ Solubilidade em água;

- KOW- coeficiente de partição octanol-água;

- $\quad$ KH - constante da Lei de Henry;

- DT50 - tempos de meia-vida no solo; e

- $\quad$ DT50 - tempo de meia-vida devido à Hidrólise.

Para a estimativa do potencial de contaminação da atrazina em águas superficiais e subterrâneas, utilizou-se a metodologia Goss (GOSS, 1992) e índice GUS (GUSTAFSON, 1989). Tais potenciais baseiam-se apenas nas propriedades físico-químicas dos compostos (KOC, DT50 no solo e solubilidade em água).

Na Figura 1 foi sistematizada a metodologia empregada para análise da ocorrência da atrazina em água e sua remoção dessa matriz. A fim de se verificar a ocorrência da atrazina em águas no Brasil buscaram-se trabalhos científicos publicados entre os anos de 2000 e 2017. Utilizaram-se termos de pesquisa, em português e inglês, como: "agrotóxicos em águas no Brasil”, “contaminação de águas no Brasil por agrotóxicos” e “atrazina em água”, em websites como Periódicos CAPES, Science Direct e Google Acadêmico. Também nesses websites procuraram-se trabalhos que tratam sobre a remoção de atrazina em água. Dentre os principais termos de busca utilizados, em português e inglês, estão: remoção de agrotóxicos em água; remoção de atrazina da água; remoção de agrotóxicos da água por oxidação; cloração da atrazina; e remoção de agrotóxicos por tratamento convencional. Foram considerados 35 trabalhos que tratam sobre a presença da atrazina em águas e 43 sobre sua remoção.

A presença de atrazina foi considerada em três diferentes matrizes: água superficial, água subterrânea e água tratada. Já a análise de sua remoção ocorreu em diferentes tipos de 
tratamento empregados, a saber: clarificação, oxidação por compostos clorados, tratamento de ciclo completo (clarificação seguida por oxidação com compostos clorados), ozonização, fotólise, processos oxidativos avançados (POA), adsorção, nanofiltração, tratamentos combinados, dentre outros.

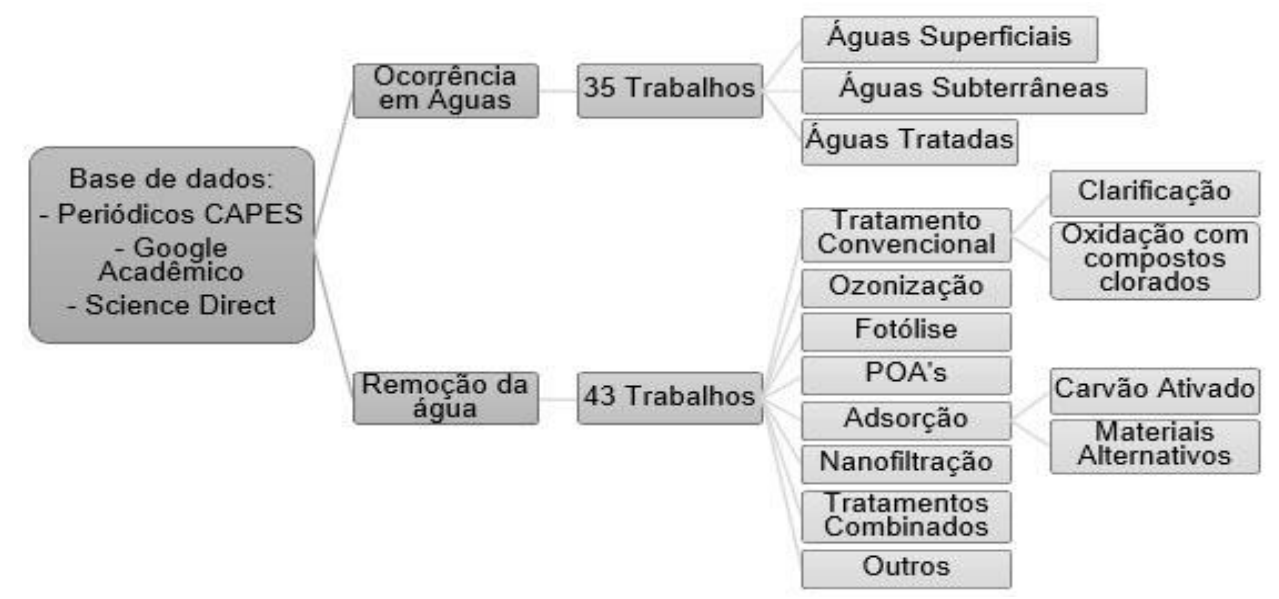

Figura 1. Sistematização da metodologia empregada para revisão bibliográfica Fonte: Autoria própria, 2018.

\section{RESULTADOS E DISCUSSÃO}

\section{Dinâmica Ambiental}

No ambiente, os agrotóxicos estão sujeitos a sofrerem reações químicas, intermediadas ou não por micro-organismos, ou interações físicas com o meio. As propriedades físicas e químicas possibilitam a análise, caracterização e previsão do possível comportamento de tais compostos no ambiente. A avaliação do comportamento pode determinar, por exemplo, a probabilidade do agrotóxico contaminar águas superficiais e subterrâneas, além de permanecer no solo ou se volatilizar.

Através dos dados na Tabela 1, pode-se inferir que a atrazina apresenta elevada probabilidade de contaminar águas subterrâneas, uma vez que é fracamente adsorvida na fração orgânica do solo (KOC - 10 a 100 mg/L) (ATSDR, 1992), o que facilita sua lixiviação. Segundo critérios estabelecidos por Rogers (1996) a atrazina ainda apresenta média hidrofilicidade $(2,5<\mathrm{KOW}<4,0)$, podendo ser facilmente lixiviada ou conduzida pelo escoamento superficial, atingindo os corpos d'água. De acordo com o tempo de meia vida no solo, o composto apresenta persistência moderada em tal matriz, tendo também um decaimento moderado devido à hidrólise (IUPAC, 2018). 
0corrência de Atrazina em águas no Brasil e remoção no tratamento da água: revisão sistemática

0 resultado do potencial de contaminação de águas superficiais e subterrâneas, respectivamente, estimado com a utilização da metodologia Goss (G0SS, 1992) e índice GUS (GUSTAFSON, 1989) está apresentado na Tabela 1. Verifica-se que o índice denota um alto risco para contaminação de águas subterrâneas. Em águas superficiais apresenta um alto potencial de contaminação para seu transporte dissolvido em água e médio para seu transporte associado ao sedimento. Tais resultados confirmam a análise das propriedades físico-químicas anteriormente realizada. Contudo, ressalta-se que para determinação desses índices não são levadas em consideração as especificidades locais, como relevo, tipo de solo e clima, que podem ter contribuições significativas na distribuição de tais compostos (SOARES et al., 2014).

Tabela 1. Propriedades da Atrazina

\begin{tabular}{|c|c|}
\hline Parâmetro & Valor \\
\hline № CAS & $1912-24-9$ \\
\hline Solubilidade em Água (mg/L) & 35 \\
\hline $\mathrm{K}_{\mathrm{oc}}$ - linear ${ }^{(1)}$ & 100 \\
\hline $\log K_{o w}^{(2)}$ & 2,7 \\
\hline $\mathrm{K}_{\mathrm{H}^{(3)}}\left(\mathrm{Pa} \mathrm{m} \mathrm{m}^{3} / \mathrm{mol}\right)$ & $1,5 \times 10^{-4}$ \\
\hline $\mathrm{DT}_{50}{ }^{(4)}$ solo (dias) & 75 \\
\hline DT $_{50}$ (4)à hidrólise (dias) & 86 \\
\hline GUS $^{(5)}$ & Alto \\
\hline Goss água(6) & Alto \\
\hline Goss sedimentos $^{(7)}$ & Médio \\
\hline
\end{tabular}

(1) KOC: Coeficiente de adsorção ao carbono orgânico do solo (IUPAC, 2018); (2) $\log$ KOW(IUPAC, 2018);(3) KH: Constante da Lei de Henry (IUPAC, 2018);(4) DT50 = tempo de meia vida(IUPAC, 2018); (5) GUS: Classificação quanto ao Índice de GUS; (6): metodologia Goss devido a dissolução em água; (7) metodologia Goss associado ao sedimento.

Fonte: Autoria própria, 2018.

\section{0corrência da Atrazina em águas superficiais, subterrâneas e tratadas no Brasil}

Na Tabela 2 é mostrada a compilação dos trabalhos considerados e seus respectivos resultados, ou seja, detecção, não deteção ou quantificação da atrazina. Ressalta-se que no caso de trabalhos em que o composto foi quantificado mais de duas vezes são apresentados apenas os valores mínimos e máximos (faixa de ocorrência). Nota-se que as regiões Sudeste, Sul e Centro-0este detêm a maior parte dos estudos, sendo apenas dois referentes à região Nordeste. Essas três regiões são também as que registraram maior quantidade de vendas do produto entre 2009 e 2016, sendo superior a 90\% considerando-se apenas os locais de venda identificados (BRASIL, 2018b). Em águas tratadas a atrazina foi quantificada nos estudos de 
Demoliner (2008) e Marques et al. (2007). Contudo, em ambos estudos, suas concentrações apresentaram-se abaixo do VMP preconizado pela PRC MS no 5 de 2017, Anexo XX (Tabela 2).

Tabela 1. Presença e faixa de ocorrência de Atrazina em Águas Superficiais, Subterrâneas e Tratadas no Brasil.

\begin{tabular}{|c|c|c|c|c|c|c|c|c|}
\hline Local & Ano & $\begin{array}{l}\text { Conc } \\
\text { A.Sup } \mu \mathrm{g} / \mathrm{L}\end{array}$ & $\begin{array}{l}\text { ConcASt } \\
\mathrm{b}^{2} \\
\mu \mathrm{g} / \mathrm{L}\end{array}$ & & $\begin{array}{l}\text { ConcATrat }{ }^{3} \\
\mu \mathrm{g} / \mathrm{L}\end{array}$ & $\begin{array}{l}\mathrm{L} Q^{4} \\
\mu \mathrm{g} / \mathrm{L}\end{array}$ & $\begin{array}{l}\mathrm{LD}^{5} \\
\mu \mathrm{g} / \mathrm{L}\end{array}$ & Fonte \\
\hline SP & $2004 / 05$ & $0,6-2,7$ & $\mathrm{NA}$ & & $\mathrm{NA}$ & 0,3 & - & Armas et al., 2007 \\
\hline SP & & $<0,02-0,06$ & $\mathrm{NA}$ & & $<0,02-0,22$ & 3,1 & 0,9 & Marques et al., 2007 \\
\hline SP & & $0,04-0,09$ & 0,03 & & $\mathrm{NA}$ & 0,02 & $\mathrm{NF}$ & Cerdeira et al., 2005 \\
\hline SP & 1999/00 & $0,02-0,52$ & $\mathrm{NA}$ & & ND & 2,92 & 0,88 & Katsuóka, 2001 \\
\hline SP & & $0,11-1,92$ & $\mathrm{NA}$ & & NA & 0,10 & 0,07 & Botelho, 2013 \\
\hline SP & & $\mathrm{NA}$ & ND & & NA & $\mathrm{NF}$ & 0,01 & Beda, 2014 \\
\hline SP & & ND & $\mathrm{NA}$ & & NA & 170 & 50 & Cappelini, 2008 \\
\hline SP & & 10,4 & 0,291 & & $\mathrm{NA}$ & $\mathrm{NF}$ & $\mathrm{NF}$ & Santos, 2013 \\
\hline MG e SP & & ND & $\mathrm{NA}$ & & ND & 0,08 & 0,023 & Pinto, 2002 \\
\hline MG & $2010 / 11$ & <LQ & $\mathrm{NA}$ & & NA & 0,010 & 0,005 & Soares, 2011 \\
\hline MG & & $<0,1$ & $<0,1$ & & NA & 0,12 & 0,0024 & Castro, 2011 \\
\hline MG & & $<\mathrm{LQ}$ & $\mathrm{NA}$ & & NA & 0,5 & & Bucci, 2015 \\
\hline MG & & $<\mathrm{LD}$ & $\mathrm{NA}$ & & $\mathrm{NA}$ & $\begin{array}{l}0,0017 \\
0,0091 \\
0,0037\end{array}$ & $\begin{array}{l}0,0005 \\
0,0027 \\
0,0011\end{array}$ & Silva, 2010 \\
\hline RJ & $2007 / 08$ & ND & $\mathrm{NA}$ & & ND & 0,2 & 0,04 & Rangel, 2008 \\
\hline RS & & $0,13-0,82$ & $0,240,6$ & & $\mathrm{NA}$ & $\mathrm{NF}$ & 0,1 & Bortoluzzi et al., 2007 \\
\hline RS & 2012 & ND & NA & & NA & 0,5 & 0,15 & Donato, 2012 \\
\hline RS & & $0,19-0,63$ & $\mathrm{NA}$ & & NA & $\mathrm{NF}$ & $<0,1$ & Gonçalves, 2007 \\
\hline RS & & ND & 0,$29 ; 0,2$ & & $\mathrm{NA}$ & NF & 0,08 & Sequinatto et al., 2006 \\
\hline RS & & $\begin{array}{l}0,0027- \\
0,0049\end{array}$ & $\mathrm{NA}$ & & 0,0043 & 0,004 & 0,004 & Demoliner, 2008 \\
\hline $\mathrm{SC}$ & & $<\mathrm{LD}$ & $\mathrm{NA}$ & & $\mathrm{NA}$ & NF & 0,1 & Mendes et al., 201l \\
\hline $\mathrm{SC}$ & & ND & $\mathrm{NA}$ & & NA & 0,57 & 0,19 & Costa et al, 2008 \\
\hline $\mathrm{SC}$ & & ND & $\mathrm{NA}$ & & NA & 5,6 & 1 & $\begin{array}{l}\text { Pinheiro, Silva e Kraisch, } \\
2010\end{array}$ \\
\hline PR & & $0,01180,302$ & $\mathrm{NA}$ & & $\mathrm{NA}$ & $\mathrm{NF}$ & $\mathrm{NF}$ & Moura, 2013 \\
\hline PR & & NA & Detec. & & $\mathrm{NA}$ & $\mathrm{NF}$ & $\mathrm{NF}$ & Schleder et al., 2017 \\
\hline G0 & & ND & $\begin{array}{l}0,02 \\
4,84\end{array}$ & - & NA & 5 & 1,6 & Rocha, 2011 \\
\hline G0/MT & & NA & $\begin{array}{l}0,001 \\
0,005\end{array}$ & - & NA & 0,01 & 0,001 & Morais, 2009 \\
\hline \multirow[t]{2}{*}{ MT } & \multirow[t]{2}{*}{$2007 / 09$} & $0,02-4,92$ & $\begin{array}{l}0,01 \\
0,02\end{array}$ & - & - & NF & NF & \multirow[t]{2}{*}{ Moreira et al., 2012} \\
\hline & & $0,25-9,33$ & 18,96 & & $\mathrm{NA}$ & $\mathrm{NF}$ & $\mathrm{NF}$ & \\
\hline MT & 2002/03 & $\mathrm{NA}$ & 0,07 & & $\mathrm{NA}$ & $\mathrm{NF}$ & 0,01 & Souza et al., 2004 \\
\hline MT & & Detec. & $\mathrm{NA}$ & & $\mathrm{NA}$ & 0,20 & 0,03 & Figueiredo, 2012 \\
\hline MT & & ND & $\mathrm{NA}$ & & NA & $\mathrm{NF}$ & $\mathrm{NF}$ & Maraschin, 2003. \\
\hline MT & 2007 & $0,006-0,048$ & $\mathrm{NA}$ & & $\mathrm{NA}$ & $\mathrm{NF}$ & 0,0026 & $\begin{array}{l}\text { Calheiros, Ferracini e } \\
\text { Queiroz, 2010 }\end{array}$ \\
\hline MT & & $0,002-0,018$ & $\mathrm{NA}$ & & $\mathrm{NA}$ & 0,002 & $\mathrm{NF}$ & Laabs et al., 2002. \\
\hline MS & 2004 & $\mathrm{NA}$ & ND & & ND & 0,25 & 0,084 & Lopes et al., 2011 \\
\hline CE & & NA & $\begin{array}{l}<\mathrm{L}, \\
9,95\end{array}$ & - & NA & 0,10 & 0,05 & Barreto, 2006 \\
\hline CE & $2003 / 04$ & $\mathrm{NA}$ & $\begin{array}{l}0,76 \\
42,77 \\
\end{array}$ & - & NA & NF & $\mathrm{NF}$ & $\begin{array}{l}\text { Barreto, } \quad \text { Araújo } \\
\text { Nascimento, } 2004 .\end{array}$ \\
\hline
\end{tabular}

1: Concentração em Águas Superficiais; 2: Concentração em Águas Subterrâneas; 3: Concentração em Águas Tratadas; 4: Limite de Quantificação; 5: Limite de Deteç̧ão. Detec.: Detectado; NA: Não Avaliado; ND: Não Detectado; NF: Não Fornecido.

Fonte: Autoria própria, 2018. 
0corrência de Atrazina em águas no Brasil e remoção no tratamento da água: revisão sistemática

Na Figura 2 é mostrada a variação dos dados de concentração da atrazina, reportados pela literatura referenciada, em águas superficiais (Figura 2.A) e subterrâneas (Figura 2.B) brasileiras. A imagem à esquerda de cada figura apresenta todos os dados (incluindo outliers) e a figura da direita mostra o boxplot dos dados com exclusão de outliers, o que facilita a visualização da dispersão dos dados. Devido à escassez de dados quantificados do composto em águas tratadas (Tabela 2), não foi possível a elaboração de gráfico para a ocorrência da atrazina em tal matriz.

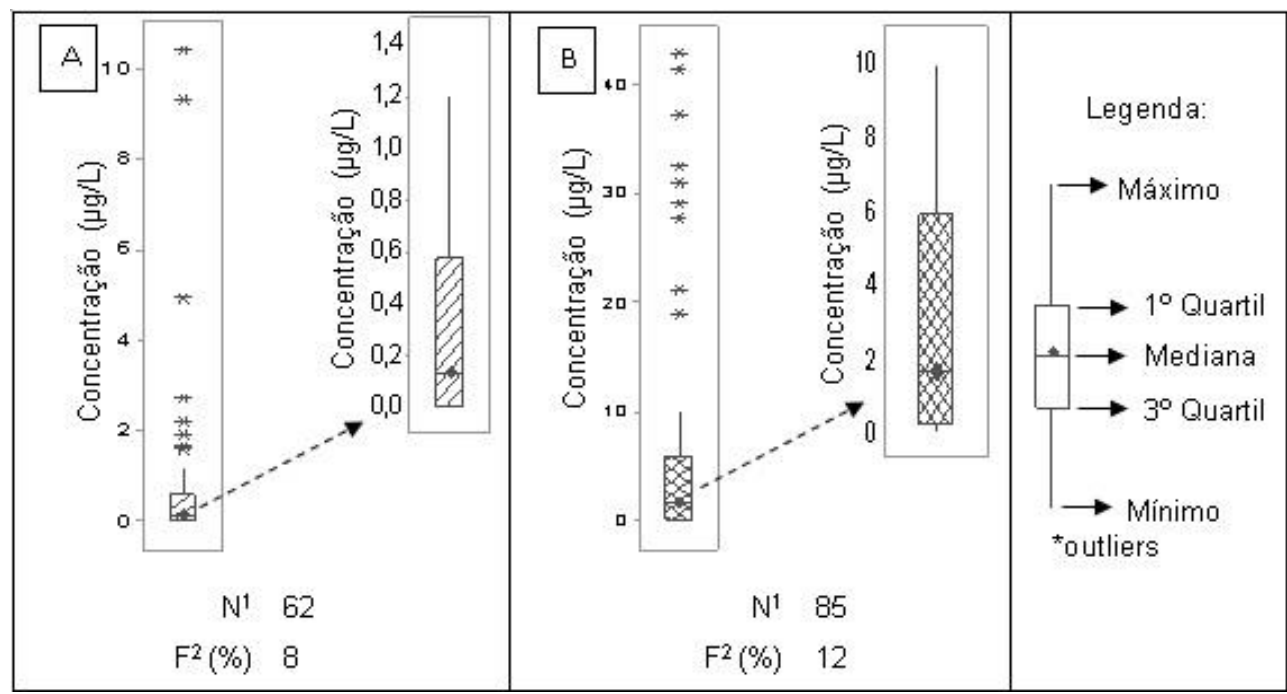

Figura 2. Ocorrência de Atrazina no Brasil.

A - Águas Superficiais. B - Águas Subterrâneas.

Nl: Número de dados considerados no gráfico; F²: Frequência de detecção e/ou quantificação.

Fonte: Autoria própria, 2018.

Segundo WHO (2017), a concentração da atrazina em água raramente excede $2 \mu \mathrm{g} / \mathrm{L}$ (VMP). No Brasil, em águas superficiais, nota-se que essa informação procede, uma vez que pelo menos $75 \%$ dos dados reportados pela literatura apresentam-se abaixo de $1 \mu \mathrm{g} / \mathrm{L}$ (Figura 2). Entretanto, há estudos que relatam concentrações acima do VMP, chegando inclusive a um

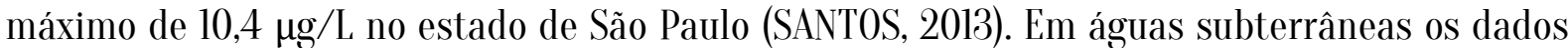
de concentração avaliados apresentaram maior amplitude comparativamente aos dados para águas superficiais (Figura 2), variando entre 1x10-6 $\mu \mathrm{g} / \mathrm{L}$ (MORAIS, 2009) a 42,8 $\mu \mathrm{g} / \mathrm{L}$ (BARRET0, ARAÚJ0 e NASCIMENT0 et al., 2004). Neste caso, ressalta-se ainda que, mais de 40\% do total de dados considerados apresentou concentrações superiores ao VMP. Esses dados corroboram a estimativa do elevado potencial de contaminação de águas subterrâneas pela 
atrazina. A frequência de detecção do composto no total de amostras analisadas nos trabalhos considerados foi de 8 e 12\%, respectivamente, para águas superficiais e subterrâneas.

\section{Remoção da Atrazina no Tratamento de Água}

Os resultados de remoção da atrazina nos processos de tratamento de água estão apresentados na Figura 3.

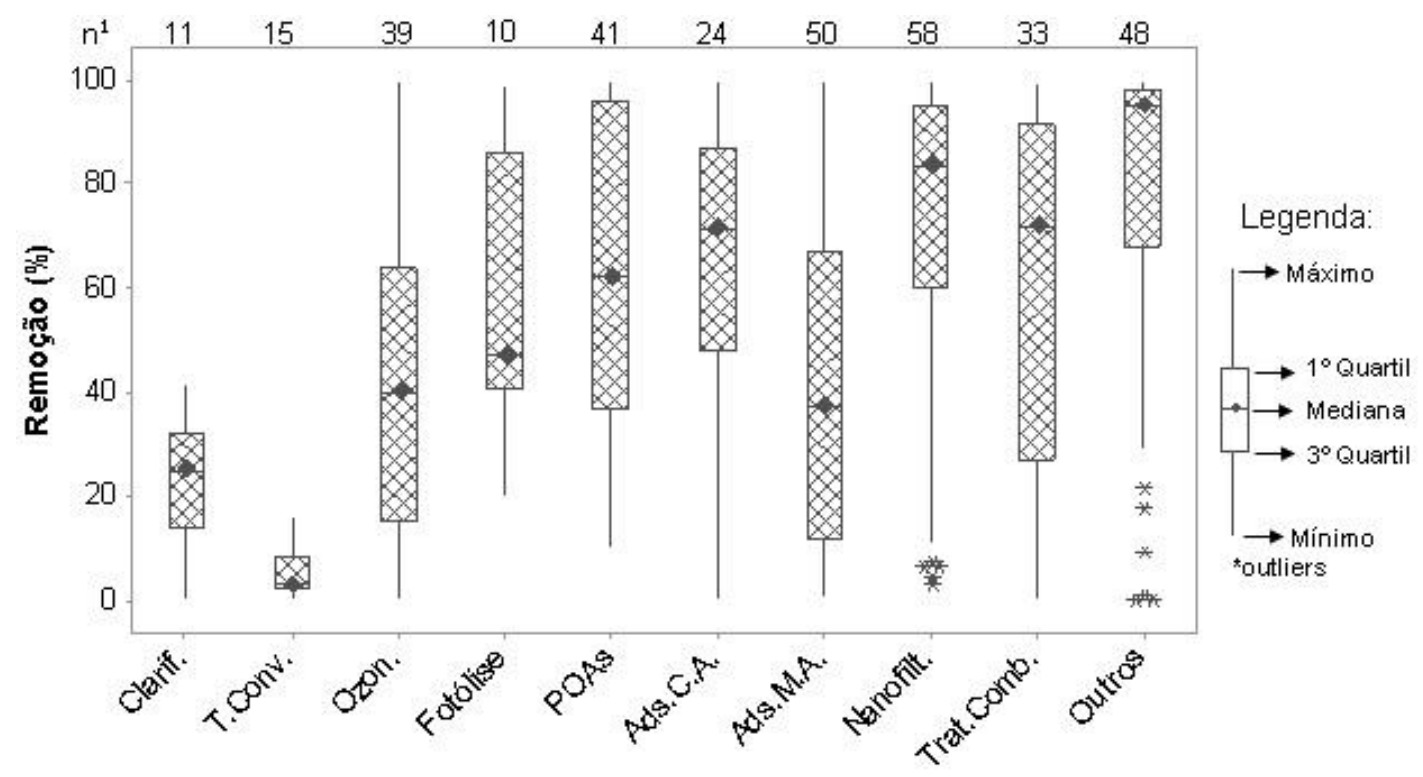

Figura 3. Remoção de Atrazina em processos utilizados no Tratamento de Água

nl: Número de dados considerados; Clarif.: Clarificação; T.Conv.: Tratamento Convencional; Ozon.: Ozonização; Ads.C.A.: Adsorção em Carvão Ativado; Ads.M.A.: Adsorção em Materiais Alternativos; Nanofilt.: Nanofiltração; Trat.Comb.: Tratamentos Combinados.

Fonte: Autoria própria, 2018.

Percebem-se remoções inferiores a 45\% associadas ao tratamento de ciclo completo, sendo essa a tecnologia mais usada no Brasil. De acordo com Thuy et al. (2008), que obteve 41\% de remoção da atrazina na etapa de clarificação, o principal mecanismo de remoção de alguns micropoluentes é através da adsorção nos colóides presentes na água. Os autores variaram a concentração do coagulante (sulfato de alumínio hidratado) entre 0 e $300 \mathrm{mg} / \mathrm{L}$, mas a maior remoção ocorreu na ausência do coagulante, possivelmente pela adsorção aos colóides da água. Ormad et al. (2008) reportaram apenas 20\% de remoção do contaminante via oxidação por cloração na concentração de 18 mg Cl2/L (ORMAD et al., 2008). Eficiências inferiores a 20\% associadas ao tratamento convencional foram obtidas por Hladik, Roberts e Bouwer (2008). De acordo com o exposto, nota-se que o tratamento de ciclo completo (clarificação + oxidação 
0corrência de Atrazina em águas no Brasil e remoção no tratamento da água: revisão sistemática

com compostos clorados) não é suficiente para a remoção de atrazina da água, ainda que em uma dose de cloro superior àquelas utilizadas em ETA (5 mg/L) (BRASIL, 2017).

Analisando-se a Figura 3, nota-se que tratamentos menos utilizados em ETA como a ozonização, P0A, nanofiltração, entre outros, podem chegar a remoções elevadas, até mesmo próximas a 100\% de eficiência. 0s dados de remoção reportados em estudos sobre a ozonização da atrazina apresentam mediana da ordem de 40\% de remoção. Contudo, eficiências superiores a $90 \%$ foram obtidas em estudos que avaliaram o processo em meio alcalino ( $\mathrm{pH}=10)$ (YIXIN et al., 2014; SILVA et al. 2010), condição na qual o processo ocorre na presença de radicais hidroxila, que são oxidantes mais fortes do que o ozônio, o que resulta em maiores eficiências comparativamente àquelas reportadas em pHs baixos ou próximos à neutralidade. Com doses de ozônio iguais a 1,6 mg/L Coelho e Di Bernardo (2017) também obtiveram remoção de 84 a 95\% e com 2,8 mg 03/L a remoção chegou a 99\%.

A fotólise com a utilização de radiação UV, comprimento de onda na faixa de 180 a 1100 nm, Baranda, Fundazurib e Maranón (2014) obtiveram remoções que variaram de 20 a 85\% (Figura 3). Sendo a maior remoção associada ao maior número de pulsos aplicados, os quais variaram entre 4, 6 e 15 pulsos (BARANDA, FUNDAZURIB e MARANÓN, 2014). Bianchi et al. (2006) obtiveram remoção superior a 99\% após 100 minutos de fotólise, com comprimento de onda de $254 \mathrm{~nm}$, tempo esse muito elevado considerando a realidade de ETA.

Processos oxidativos avançados (POA) como a ozonização catalítica, foto-Fenton e Eletron-Fenton foram estudados para a remoção de atrazina da água. Na Figura 3 percebe-se que apesar de apresentar uma remoção máxima de 100\%, a mediana desses processos foi inferior a 65\% de remoção. Com a ozonização catalisada por peróxido de hidrogênio, Snyder et al. (2006), variando o tempo de reação $(2,4,14$ e 24 min) e concentração dos reagentes, obtiveram uma faixa de remoção entre 19 e 62\%. Menores eficiências foram referentes a menor dose de ozônio e tempos de contato. Yixin et al. (2014), com a ozonização catalisada com dióxido de Titânio, a eficiência de remoção variou aproximadamente entre 40 e $98 \%$ em função do pH, sendo o aumento desse acarretou no aumento da remoção. Balci et al. (2009) e Kassinos et al. (2009), obtiveram resultados satisfatórios com remoção de até 100\% em tempos de reação de 30 min, pH de 3 e 2,5 utilizando os processos eletro Fenton e foto Fenton. Nesse caso a desvantagem ocorre devido ao pH baixo o qual não é comumente utilizado em ETA.

A adsorção em carvão ativado se mostra mais eficiente em relação a adsorção utilizando materiais alternativos, com medianas de $70 \%$ e $40 \%$ respectivamente (Figura 3). A adsorção mostrou-se eficaz para a remoção do composto atingindo níveis acima de 90\% para 
adsorventes como o carvão ativado em pó - CAP com TC de 2 h (ZHOU et al., 2014), apesar da atrazina apresentar baixo KOC e KOW (Tabela 1). Materiais alternativos como microesfera magnética (ZHOU et al., 2014) e argila montmorilonita com polímero (ZADAKA et al., 2009) também reportaram remoções superiores a 90\%. Eficiências com amplitude de 5 a 85\% foram obtidas por Humbert et al. (2008), mantendo uma alta $\mathrm{Ci}(200 \mu \mathrm{g} / \mathrm{L})$ e variando o tipo de adsorvente e TC. A maior remoção está associada a um TC de 24 h e a utilização de CAP. Ao utilizar uma Ci de $1 \mu \mathrm{g} / \mathrm{L}$ e TC fixo em 30 min, a faixa de remoção foi entre 5 e 90\%. Sendo que a remoção por CAP em água deionizada reportou a maior remoção, enquanto em água bruta foi da ordem de 58\% indicando assim a interferência causada pela matéria orgânica presente nessa matriz (HUMBERT et al., 2008). Já com a utilização da membrana AMBERSORB (8 mL/L) alcançou remoção da ordem de 85\% (HUMBERT et al., 2008). A OMS reporta que uma concentração final de $0,1 \mu \mathrm{g} / \mathrm{L}$ do composto pode ser alcançada com adsorção em carvão ativado granular e CAP e nanofiltração (WH0, 2017).

Remoção de até 98\% foi reportada para o tratamento utilizando nanofiltração (LAINE,1997 apud RIBEIR0 e LUCA,1998), mesmo resultado máximo obtido por Hofman et al. (1997, apud USEPA 2001), utilizando o mesmo processo. Caus et al. (2009) obtiveram remoções do composto superiores a $98 \%$ com o processo de nanofiltração com e sem recirculação, utilizando dois tipos diferentes de membranas (Desal51HL e NF270). De acordo com a Figura 3, pode-se notar que as remoções por nanofiltração apresentam eficiências medianas superiores a $80 \%$ e valor máximo de $99,7 \%$, o que indica a viabilidade do processo para a remoção de atrazina, contudo, devem-se estabelecer as melhores condições para sua utilização. Uma vez que eficiências inferiores a 20\% também foram reportadas por Sanches et al. (2013), Kiso et al. (2000), Caus et al. (2009) e Berg, Hagmeyer e Gimbel (1997) para as membranas DK2540F1073, NTR-7450, NTR-7410, NF30 e NFCA50.

Associação de tratamentos também pode ser uma boa opção para a remoção de atrazina da água. Ao se utilizar CAP, WHP Calgon e Hydrodarco,em conjunto com o tratamento de ciclo completo, Miltner et al. (1989 apud USEPA, 2001), obtiveram remoção de até 87\%. 0 aumento da dose de CAP (2,8 a $33 \mathrm{mg} / \mathrm{L})$ resultou no aumento da eficiência do processo. 0 sucesso do processo foi atribuído pelos autores à etapa de adsorção devido à comprovação prévia da ineficiência do tratamento convencional (MILTNER et al., 1989 apud USEPA, 2001). Tratamentos de pré-oxidação, tanto com composto clorado quanto com ozônio, seguido por adsorção+coagulação removeram 90\% do composto (ORMAD et al., 2008). 
0corrência de Atrazina em águas no Brasil e remoção no tratamento da água: revisão sistemática

Outros tratamentos também podem ser utilizados a fim de remover o composto da água, dentre os quais se encontra a osmose inversa, capaz de remover até 100\% ao se utilizar membrana um filme composto fino (FT-30 e DSI) (FR0NK e BAKER, 1990 apud USEPA, 2001). A utilização de filtros lentos em areia e carvão ativado também apresentaram remoções superiores a 90\% (ZANINI, 2010). A tabela 3 possui uma compilação das faixas de remoção da atrazina para cada tratamento.

Tabela 3. Faixas de remoção da atrazina por tratamento (\%).

\begin{tabular}{llllllllllll}
\hline Trat. & Clarif & Clor & T.Con & Ozon & Foto & POA & Ads.C. & Ads.M. & Nano & T.Com & Outro \\
\hline$\%$ & $0-41$ & 20 & $1-16$ & $0-100$ & $20-99$ & $10-100$ & $0-100$ & $1-99,5$ & $3-99,7$ & $0-99$ & $0-100$ \\
\hline
\end{tabular}

Trat:: Tratamentos; Clarif.: Clarificação; Clor.: Cloração; T.Con.: Tratamento Convencional; Ozon.: Ozonização; Foto.: Fotólise; P0A: Processos 0xidativos Avançados; Ads.C..: Adsorção em Carvão Ativado; Ads.M.: Adsorção em Materiais Alternativos; Nano.: Nanofiltração; T.Com.: Tratamentos Combinados.

Fonte: Autoria própria, 2018.

\section{Subprodutos da Atrazina}

Os subprodutos da atrazina mais comumente encontrados no ambiente são desetilatrazina - DEA, desisopropilatrazina - DIA, didealquilatrazina - DDA, desetilhidroxiatrazina - DEHA e hidroxiatrazina - HA (LOOS; NIESSNER, 1999 apud COELHO; DI BERNARD0, 2017), suas estruturas químicas estão apresentadas na Figura 4. Segundo WH0 (2017) a toxicidade e modo de ação dos metabólitos cloro-s-triazina no organismo humano são semelhantes ao da atrazina, a exceção do HA que apresenta toxicidade para os rins (WHO, 2017).Vale ressaltar que o HA é o principal subproduto da degradação da atrazina por fotólise (BIANCHI et al., 2006).

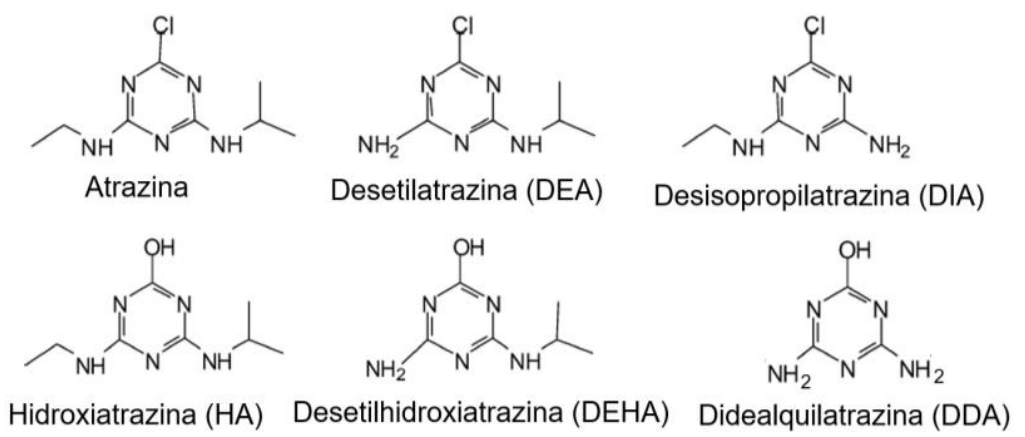

Figura 4. Estrutura química da Atrazina e de seus principais subprodutos. Fonte: Adaptado de: Salomon et al. (2008); Jiang; Adams (2006). 
0 anel s-triazina apresenta resistência à oxidação pelo radical hidroxila H0 (HUSTON; PIGNAATELO, 1999) gerado nos P0A. Os principais mecanismos de degradação do composto por P0A são desalquilaminação e descloração do anel até a obtenção do ácido cianúrico, subproduto final da atrazina nestes processos (KEARNEY et al., 1988 apud HUSTON; PIGNATELO, 1999; PELIZZETTI et al., 1990 apud HUSTON; PIGNATELO, 1999). Yixin et al. (2014) identificou cinco produtos da degradação da atrazina no processo de ozonização catalítica com Ti02, sendo eles: DEA, DIA, desetil-desisopropil-atrazina (DEIA), diaminotriazina (DAA) e 5- azauracil (DOA).

Jiang e Adams (2006) estudaram a remoção da atrazina da água por ozonização e formação de seus metabólitos DEA, DIA e DDA. Os autores identificaram o DEA como o produto inicial da degradação da atrazina por ozonização em meio aquoso e pH 6 e 9, com posterior formação do DIA. Não se observou a formação de DDA nas condições empregadas $(0-5 \mathrm{mg} / \mathrm{L}$ de ozônio), mas os autores acreditam que haveria a formação caso fosse empregada dose mais elevada do oxidante. Em estudos realizados por Coelho e Di Bernardo (2017) envolvendo o monitoramento da formação de DEA, DIA e DEHA durante a ozonização da atrazina em concentração inicial entre 78,9 e 110,2 $\mu \mathrm{g} / \mathrm{L}$, verificou-se que a aplicação de 2,8 mg/L de ozônio, apesar de resultar em concentração efluente de atrazina inferior a $2 \mu \mathrm{g} / \mathrm{L}$ após de 12 minutos de ozonização, o somatório dos subprodutos foi 3,94 $\mu \mathrm{g} / \mathrm{L}$, o que acarretaria problemas sanitários para a utilização dessa água já que esses compostos apresentam efeitos tóxicos semelhantes ao da atrazina (COELHO e DI BERNARDO, 2017). Os autores recomendam o monitoramento do DEA, por ser o principal subproduto da ozonização da atrazina, em águas de abastecimento e sua inclusão em futuras revisões do padrão de potabilidade da água no Brasil devido à similaridade toxicológica com o seu precursor.

\section{CONCLUSÃO}

0 presente estudo evidencia que águas superficiais e subterrâneas são passíveis de serem contaminadas pela atrazina. No Brasil, há relatos de sua ocorrência em níveis superiores ao VMP, estabelecido pela PRC MS n ${ }^{0} 5$ de 2017, Anexo XX em ambas as matrizes. Contudo, as maiores concentrações, bem como a frequência de deteç̧ão foram reportadas em estudos que avaliaram águas subterrâneas. A avaliação dos estudos contemplados mostra que 0 tratamento convencional é ineficaz na remoção da atrazina em ETA. Desta forma, recomenda- 
0corrência de Atrazina em águas no Brasil e remoção no tratamento da água: revisão sistemática

se manter o monitoramento de tal composto na água tratada assim como de seus subprodutos. Tratamentos avançados com eficiência comprovada em diversos estudos são necessários. Dentre os processos recomendados para garantia da remoção do contaminante durante o tratamento de águas em ETA citam-se: nanofiltração, adsorção em carvão ativado e osmose inversa. A combinação de tratamentos também deve ser considerada uma vez que estudos que contemplam oxidação+coagulação+adsorção mostraram excelentes resultados. Além disso, os P0A também merecem destaque, uma vez que, em geral, estudos envolvendo tais processos apresentaram bons resultados. Contudo deve-se atentar para a viabilidade técnica, econômica e possibilidade de geração de subprodutos tóxicos durantes esses processos.

\section{AGRADECIMENT0}

À Pró-reitoria de Pesquisa e Pós-Graduação da UFJF. À Fundação de Amparo à Pesquisa do Estado de Minas Gerais- FAPEMIG.

\section{REFERÊNCIAS BIBLIOGRÁFICAS}

ARMAS, E. D.; MONTEIR0, R. T. R.; ANTUNES, P. M.; SANTOS, M. A. P.; CAMARG0, P. B. Diagnóstico Espaço-Temporal Da 0corrência De Herbicidas Nas Águas Superficiais Esedimentos Do Rio Corumbataí E Principais Afluentes. Quimica Nova. v.30, n.5, p.1119-1127, 2007.

ATSDR - Agency for Toxic Substances \& Disease Registry. ATSDR public health assesment guidance manual. 1992. Disponível em: https://books.google.com.br/books?id=eF6_mn0xcHcCEpg=PAll\&lpg=PAll\&dq=atsdr++Koc\&source=bl\&ots=epvp8fxHHEGsig=bU0Z4eosSMhpUVv64_H14XbvV20\&hl=pt-

BREsa=X\&ved=0ahUKEwi024cDXAhVCGpAKHTF8BRQQ6AEISTAE\# $\mathrm{v}=$ onepage\&q=atsdr\%20-\%20Koc\&f=false.

Acessado em 27 mar. 2018.

BAIRD, C.; CANN, M. Química Ambiental. 4 ed. Bookman, 2011.

BALCI, B.; OTURAN, N.; CHERRIER, R.; OTURAN, M. A. Degradation of atrazine in aqueous medium by electrocatalytically generated hydroxyl radicals. A kinetic and mechanistic study. Water research, v. 43, n. 7, p. 1924-1934, 2009.

BARANDA, A. B.; FUNDAZURIB, 0; MARANÓN, I. Photodegradation of several triazidic and organophosphoruspesticides in water by pulsed light technology. Journal of Photochemistry and Photobiology A: Chemistry, n. 286, p. 29-39, 2014.

BARRET0, F. M. D. S.; ARAÚJ0, J. C. D.; NASCIMENT0, R. F. D. Caracterização Preliminar da Carga de Agrotóxico Presente na Água Subterrânea em Tianguá - Ceará (BRASIL). In: Congresso Brasileiro de Águas Subterrâneas, 13. São Paulo: 20 p. 2004.

BARRETO, F. M. D. S. Contaminação da Água Subterrânea por pesticidas enitrato no Município de Tianguá, Ceará. 2006. 166p. Tese (Dutorado em Engenharia Civil) Departamento de Engenharia Hidráulica e Ambiental, Universidade Federal do Ceará, Fortaleza. 
BEDA, C. Avaliação de Agrotóxicos de uso canavieiro em águas subterrâneas: uma proposta para oaquífero Guarani. 2014. 96p. Dissertação (Mestrado em Ciências). Escola de Enfermagem de Ribeirão Preto, Universidade De São Paulo, Ribeirão Preto, SP.

BERG, P.; HAGMEYER, G.; GIMBEL, R. Removal of pesticides and other micropollutants by nanofiltration. Desalination, v. 113, n. 2-3, p. 205-208, 1997.

BIANCHI, C. L.; PIROLA, C.; RAGAINI, V.; SELLI, E. Mechanism and efficiency of atrazine degradation under combined oxidation processes. Applied Catalysis B: Environmental, v. 64, n. 1-2, p. 131-138, 2006.

BORTOLUZZI, E. C.; RHEINHEIMER, S. D.; GONÇAlVES, C. S.; PELLEGRINI, J. B. R.; MARONEZE, A. M.; KURZ, H. S.; BACAR, N. M.; ZANELLA,R. Investigation of the 0ccurrence of Pesticide Residues in Rural Wells and Surface Water Following Application to Tobacco. Química Nova, v.30, p. 1872-1876, 2007.

BOTELHO, R. G. Avaliação da qualidade da água do rio Piracicaba (SP) e efeito da vinhaça para organismos aquáticos antes e após a correção do pH. 2013. 110p. Tese (Doutorado em Ciências). Centro de Energia Nuclear na Agricultura da Universidade de São Paulo, Universidade de São Paulo, Piracicaba, SP.

BRASIL. MMA - Ministério do Meio Ambiente. Disponível em: http://www.mma.gov.br/segurancaquimica/agrotoxicos. Acessado em 27 de março de 2018. (a)

IBAMA - Instituto Brasileiro do Meio Ambiente. Disponível em: http://www.ibama.gov.br/agrotoxicos/relatorios-de-comercializacao-de-agrotoxicos. Acessado em 25 de março de 2018. (b)

ANVISA - Agência Nacional de Vigilância Sanitária. Disponível em: http://portal.anvisa.gov.br/documents/111215/117782/a14.pdf/76773817-be41-4334-9846-77bd559f9e80. Acessado em 27 de março de 2018. (c)

Lei 7.802, de 11 de julho de 1989. Diário Oficial [da] República Federativa do Brasil, Poder Executivo, Brasília, DF, 11 jul. 1989.

Decreto $\mathrm{n}^{\circ}$ 4.074. Regulamenta a Lei no 7.802, de 11 de julho de 1989, que dispõe sobre a pesquisa, a experimentação, a produção, a embalagem e rotulagem, o transporte, o armazenamento, a comercialização, a propaganda comercial, a utilização, a importação, a exportação, o destino final dos resíduos e embalagens, o registro, a classificação, o controle, a inspeção e a fiscalização de agrotóxicos, seus componentes e afins, e dá outras providências, 2002.

. MINISTÉRIO DA SAÚDE. Portaria de Consolidação nº 5, de 28 de setembro de 2017 - ANEX0 XX, Brasília, DF, 03 out. 2017. Seção 1, p. 360.

BUCCI, M. M. H. S. Estudo da qualidade da água na represa Dr. João Penido: metais, agrotóxicos, índices de qualidade da água e de estado trófico (Juiz de Fora, MG). 2015. 163p. Tese (Doutorado em Ecologia Aplicada ao Manejo e Conservação de Recursos Naturais). Programa de Pós-Graduação em Ecologia, Universidade Federal de Juiz de Fora, Juiz de Fora, MG.

CALHEIROS, D. F.; FERRACINI, V. L.; QUEIR0Z, S. C. N. Contaminação por agrotóxicos nas águas da Bacia do Alto Paraguai. In: Seminário de Agroecologia de Mato Grosso do Sul, 3 e Encontro de Produtores Agroecológicos de MS. 2010, Corumbá, MS. Construindo um Futuro Sustentável e Solidário. p. 1-5.

CAPPELINI, L. T. D. Análise dos pesticidas ametrina, atrazina, diuron e fipronil em amostras de água do Ribeirão do Feijão - São Carlos- SP. 2008. 69p. Dissertação (Mestrado em Ciências). Instituto de Química de São Carlos, Universidade de São Paulo, São Carlos, SP.

CARM0, D. A.; CARM0, A. P. B.; PIRES, J. M. B.; OLIVEIRA, J. L. M. Comportamento ambiental e toxidade dos herbicidas atrazina e simazina. Ambi-Agua, Taubaté, v. 8, n. 1, p. 133-143, 2013. 
CASTR0, E. D. Estudo Hidrogeoquímico Ambiental em Região Cárstica no Município Sete Lagoas - Minas Gerais: Investigação da Contaminação de Águas Superficiais e Subterrâneas por Nitrato e Atrazina, na Área do Centro Nacional de Pesquisa de Milho e Sorgo da EMBRAPA. 2011.890p. Tese (Doutorado em Saneamento, Meio Ambiente e Recursos Hídricos). Universidade Federal de Minas Gerais, Belo Horizonte, Minas Gerais.

CAUS, A., VANDERHAEGEN, S., BRAEKEN, L., \& VAN DER BRUGGEN, B. Integrated nanofiltration cascades with low salt rejection for complete removal of pesticides in drinking water production. Desalination, v. 241, n. 1-3, p. 111-117, 2009.

CERDEIRA, A. L.; PESSOA, M. C. P. Y.; SANTOS, N. A. G.; LANCHOTE, V. L. Lixiviação de atrazina em solo em área de recarga do aquífero guarani. Revista Brasileira de Herbicidas, v. 4, n. 2, p. 92-101, 2005.

COELHO, E .R. C.; DI BERNARD0, L. Presença e formação Atrazina e os subprodutos e Desetilatrazina, Desisopropilatrazina e Desetilhidroxiatrazina em sistema combinado de ozonização e filtração lenta. Engenharia Sanitária e Ambiental, v. 22, n. 4, 2017.

COSTA, L. L. F.; SANT’ANA, E. S.; SUCHARA, E. A.; BENATO, V. S.; CARASEK, E. Determinação de herbicidas usados no cultivo de arroz irrigado na região sul do estado de Santa Catarina através da SPME-GC-ECD. Química Nova, São Paulo, v. 31, n. 1, p.79-83, 2008.

DEMOLINER A. (2008). Otimização e validação de metodologia analítica empregando SPE e LC-ESI-MS/MS para determinação de multiclasses de agrotóxicos e metabólitos em água de superfície e de abastecimento público. 2008. 11lp. Dissertação (Mestrado em Química). Universidade Federal do Rio Grande, Rio Grande, RS.

DONAT0, F. F. Resíduos De Agrotóxicos Em Água Potável Usando Spe E Determinação Rápida Por Lc-Ms/Ms GcMs/Ms. 2012. 148p. Dissertação (Mestrado em Química). Centro De Ciências Naturais e Exatas, Universidade Federal De Santa Maria, RS.

DOS SANTOS, K. C.; MARTINEZ, C. B. R. Genotoxic and biochemical effects of atrazine and Roundup®, alone and in combination, on the Asian clam Corbicula fluminea. Ecotoxicology and environmental safety, v. 100, p. 7-14, 2014.

EUROPEAN UNION. Council Directive 98/83/EC of 3 November 1998 on the quality of water intended for human consumption. Disponível em: <http://eur-lex.europa.eu/legal-content/PT/TXT/PDF/?uri=CELEX:01998L008320151027Gfrom=EN>. Acessado em 14 mar. 2018.

FIGUEIRED0, S. B. Avaliação da Qualidade da Água da Sub-Bacia do Rio Cuiabá-MT Aplicando Análise Multivariada. 2012. 140p. Dissertação (Mestrado em Recursos Hídricos). Universidade Federal de Mato Grosso, Cuiabá, MT.

FRONK, C. A.; BAKER, D. Pesticide Removal by Membrane Processes. In: ANNUAL CONFERENCE OF AWWA,1990, Cincinnati, 0H: AWWA, 1990.

GONCALVES, C. S. Caracterização de sedimentos e de contaminantes numa microbacia hidrográfica antropizada. 2007. 160p. Tese (Doutorado em Ciência do Solo). Universidade Federal de Santa Maria, Rio Grande do Sul.

G0SS, D. W. Screening Procedure for Soils and Pesticides for Potential Water Quality Impacts. Weed Technology, v. 6, p. 701-708, 1992.

GUSTAFSON, D. I. Groundwater Ubiquity Score: a Simple Method for Assessing Pesticide Leachability. Environmental Toxicology and Chemistry, v. 8, p. 339-357, 1989.

HEALTH CANADA. 2017. Guidelines for Canadian Drinking Water Quality-Summary Table. Water and Air Quality Bureau, Healthy Environments and Consumer Safety Branch, Health Canada, Ottawa, Ontario. Disponível em: https://www.canada.ca/content/dam/hc-sc/migration/hc-sc/ewh-semt/alt_formats/pdf/pubs/watereau/sum_guide-res_recom/sum_guide-res_recom-eng.pdf. Acessado em: 24 mar. 2018.

HLADIK, M. L.; ROBERTS, A. L.; BOUWER, E. J. Neutral degradates of chloroacetamide herbicides: Occurrence in drinking water and removal during covencional water treatment. Water Reserach, v. 42, p. 4905-4914, 2008. 
HOFMAN, J. A. M. H.; BEERENDONK, E . F.; FOLMER, H. C.; KRUITHOF, J. C. Removal of Pesticides and Other Micropollutants with Cellulose-acetate, Polyamide and Ultra-low Pressure reverse 0smosis Membranes. Desalination, v. 113, p. 209-214, 1997.

HUMBERT, H.; GALLARD, H.; SUTY, H.; CROUÉ, J-P. Natural organic matter (NOM) and pesticides removal using a combination of ion exchange resin and powdered activated carbon (PAC). Water Research, v. 42, p. $1635-1643$, 2008.

HUSTON, P. L.; PIGNATELLO, J. J. Degradation of selected pesticide active ingredients and commercial formulations in water by the photo-assisted Fenton reaction. Water Research, v. 33, n. 5, p. 1238-1246, 1999.

INTERNATIONAL UNION OF PURE AND APLIED CHEMISTRY (IUPAC). Disponível em: <https://sitem.herts.ac.uk/aeru/iupac/Reports/43.htm〉. Acessado em 11 mar. 2018.

JIANG, H.; ADAMS, C. Treatability of chloro-s-triazines by conventional drinking water treatment Technologies. Water Research, v. 40, p. 1657-1667, 2006.

JOWA, L.; HOWD, R. Should atrazine and related chlorotriazines be considered carcinogenic for human health risk assessment? J Environ Sci Health C Environ Carcinog Ecotoxicol Rev., v. 29 (2), p. 91-144, 2011.

KASSINOS, D.; VARNAVA, N; MICHAEL, C.; PIERA, P. Homogeneous oxidation of aqueous solutions of atrazine and fenitrothion through dark and photo-Fenton reactions. Chemosphere, v. 74, n. 6, p. 866-872, 2009.

KATSUÓKA, L. Avaliação do impacto da atividade agropecuária na qualidade da água em áreas de captação superficial nas bacias hidrográficas dos rios Mogi-Guaçu e Pardo, São Paulo. 2001. Tese de Doutorado (Pesquisas Energéticas e Nucleares), Autarquia Associada à Universidade de São Paulo, São Paulo.

KEARNEY, P.C.; MULDOON, M.T.; SOMISH, C.J.; RUTH, J.M.; VOADEN, D.J. Biodegradation of ozonated atrazine as a wastewater disposal system. J. agric. Food chem. v. 36, n. 6, p. 1301-1306, 1988.

KISO, Y.; NISHIMURA, Y.; KITA0, T.; NISHIMURA, K. Rejection properties of non-phenylic pesticides with nanofiltration membranes. Journal of Membrane Science, v.171, p.229-237, 2000.

LAABS, V.; AMELUNG, W.; PINTO, A. A.; WANTZEN, M.; SILVA, C. J.; ZECH, W. Pesticides in surface water, sediment, and rainfall of the northeastern pantanal basin, Brazil. Journal of Environmental Quality, v.31, p.1636-1648, 2002.

LAINE, J.M.; 1997. Membrane Technology and its application to drinking water production. International Conference on water quality. Rio Grande, Brasil.

LOPES, M. N. T.; NETTO, P. T.; LOURENCETTI, C.; MARCHI, M. R. R.; RIBEIR0, M. L. Validação e aplicação de método para análise de pesticidas em água para consumo humano de dourados (MS) por CLAE/UV e CG/DTE. Revista Ecotoxicologia e Meio Ambiente, Curitiba, v.21, p. 103-116, jan/dez. 2011.

LOOS, R.; NIESSNER, R. Analysis of atrazine, terbutylazine and their N-dealkylated chloro and hydroxy metabolites by solid-phase extraction and gas chromatography-mass spectrometry and capillary electrophoresis-ultraviolet detection. Journal of chromatography A, v. 835, n. 1-2, p. 217-229, 1999.

MARASCHIN, L. Avaliação do Grau de Contaminação por Pesticidas na Água dos Principais Rios Formadores do Pantanal Mato-Grossense. 2003. 88P. Dissertação (Mestrado em Saúde e Ambiente). Universidade Federal do Mato Grosso, Cuiabá, MT.

MARIN-MORALES, M. A.; VENTURA-CAMARG0, B. D. C.; HOSHINA, M. M. Toxicity of herbicides: impact on aquatic and soil biota and human health. In: Herbicides-Current research and case studies in use. InTech, 2013.

MARQUES M. N.; COTRIM, M. B.; PIRES, M. A. F.; FILHO, 0. B. Avaliação do impacto da agricultura em áreas de proteção ambiental, pertencentes à bacia hidrográfica do rio ribeira de iguape, São Paulo. Química Nova, v. 30, n. 5, p. 1178 - 1178, 2007. 
MENDES, B. G.; BUDZIAK, D.; STOLBERG, J.; PEIXER, Z. I.; DALMARCO, J. B.; SIMIONATO, E. L.; PEDROSA, R. C.; FELIPE, K. B.; OGAWA, J.; PEGORAR0, C.; SCHEFFER, L.; BESEN, M. R.; OLIVEIRA, L. J. G. G.; GEREMIAS, R. Estudo da qualidade das águas do Rio Marombas (SC/Brasil), utilizando parâmetros físico-químicos e bioensaios. RCA. Revista de Ciências Ambientais (UniLASALLE), v. 5, N. 2, p. 43-58, 2011.

MILTNER, R. J.; BAKER, D. B.; SPETH, T. F.; FR0NK, C. A. 1989. Treatment of Seasonal Pesticides in Surface Waters. Journal (American Water Works Association), p. 43-52, 1989.

MINISTRY OF HEALTH. Guidelines for Drinking-water Quality Management for New Zealand. (3rd edn). Wellington: Ministry of Health. 2017. Disponível em: http://www.health.govt.nz/publication/guidelines-drinking-waterquality-management-new-zealand. Acessado em: 16 out. 2017.

MORAIS, L. S. R. Desenvolvimento e validação de métodos para a determinação de agrotóxicos em água e solo das áreas de recarga do aquífero guarani, na região das nascentes do rio Araguaia, MT/G0. 2009. 132p. Tese (Doutorado em Ciências). Universidade Estadual de Campinas, Campinas, SP.

MOREIRA, J. C.; PERES, F.; SIMÕES, A. C.; PIGNATI, W. A.; DORES, E. C.; VIEIRA, S. N.; STRÜSSMANN, C.; MOTT, T. Contaminação de águas superficiais e de chuva por agrotóxicos em uma região do estado do Mato Grosso. Ciência \& Saúde Coletiva. v. 17,p.557-1568, 2012.

MOURA, E. R. R. Determinação de Pesticidas no Rio Piquiri po LC/MS/MS. 2013. 88p. Dissertação (Mestrado em Ciências e Tecnologia Ambiental). Universidade Tecnológica Federal do Paraná, Curitiba, PR.

NHMRC, NRMMC (2011). Australian Drinking Water Guidelines. Paper 6 National Water Quality Management Strategy. National Health and Medical Research Council, National Resource Management Ministerial Council, Common wealth of Australia, Canberra.

ORMAD, M. P.; MIGUEL, N.; CLAVER, A.; MATESANZ, M.; OVELLEIR0, J. L. Pesticides removal in the process of drinking water production. Chemosphere, v. 71, p. 97-106, 2008.

PATUSSI, C.; BÜNDCHEN, M. Avaliação in situ da genotoxicidade de triazinas utilizando o bioensaio Trad-SHM de Tradescantia clone 4430. Ciência \& Saúde Coletiva, v. 18, p. 1173-1178, 2013.

PEliZZETTI, E.; MAURINO, V.; MINER0, C.; CARLIN, V.; TOSATO, M.L.; PRAMAURO, E.; ZERBinATI, O.; TOSATO, M.L. Photocatalytic degradation of atrazine and other s-triazine herbicides. Environmental science \& technology, v. 24, n. 10, p. 1559-1565, 1990.

PINHEIR0, A.; SILVA, M. R.; KRAISCH, R. Presença de pesticidas em águas superficiais e subterrâneas na bacia do Itajaí, SC. REGA - v. 7, n. 2, p. 17-26, 2010.

PINT0, G. M. F. Desenvolvimento de metodologia para determinação de multirresíduos de herbicidas e seus metabólitos em água e em solo por cromatografia líquida de alta eficiência. 2002. 196p. Tese (Doutorado). Universidade Estadual de Campinas, Campinas, SP.

RANGEL, C. D. F. Monitoramento de Agrotóxicos em Águas Brutas e Tratadas Destinadas ao Consumo Humano, Utilizando Método Multi-Resíduo por EFS/CG-EM. 2008. 130p. Dissertação (Mestrado). Escola Nacional de Saúde Pública, Rio de Janeiro, RJ.

RIBEIR0, M.L.; LUCA, S.J.D. Tratamento de águas por filtração por membranas. Estado da Arte. Procedings of XXVI Congresso Interamericano de Ingeneric Sanitaria y Ambiental., Lima-Peru, 1998.

ROCHA, A. A. Monitoramento de agrotóxicos em áreas irrigadas por pivô central na microbacia do Tijunqueiro, município de Morrinhos, Goiás. 2011. 145p. Tese (Doutorado em Ciências). Universidade de São Paulo, Piracicaba, SP.

ROGERS, H. R. Sources, behavior and fate of organic contaminants during sewage treatment and in sewage sludges. The Science of the Total Environment, v. 185, n. 1-3, p. 3-26, 1996. 
SANCHES, S.; PENETRA, A.; RODRIGUES, A.; CARDOSO, V. V.; FERREIRA, E.; BENOLIEL, M. J.; BARRETO CRESPO, M. T.; CRESP0, J. G.; PEREIRA, V. J. Removal of pesticides from water combining low pressure UV photolysis with nanofiltration. Separation and Purification Technology, v. 115, p. 73-82, 2013.

SANTOS, E. A. Contaminação por herbicidas em corpos hídricos da microbacia do Córrego Rico (SP) e aspectos toxicológicos de atrazine a juvenis de Piaractus mesopotamicus. 2013. 83p. Tese (Doutorado em Agronomia) Universidade Estadual Paulista, Jaboticabal, SP.

SCHLEDER, A. A.; VARGAS, L. M. P.; HANSEL, F. A.; FROEHNER, S.; PALAGANO, L. T.; ROSA FILHO, E. F. D. Evaluation of occurrence of NO3-, Coliform and atrazine in a karst aquifer, Colombo, PR. RBRH, v. 22, 2017.

SEQUINATTO, L.; REICHERT, J. M.; REINERT, D. J.; RHEINHEIMER, D. S.; COPETTI, A. C. C. Contaminação da água por agrotóxicos numa microbacia cultivada com fumo. In: XVI REUNIÃO BRASILEIRA DE MANEJ0 E CONSERVAÇÃO DO SOLO E DA ÁGUA, 2006, Aracajú, SE. Novos desafios do carbono no manejo conservacionista. Aracajú, SE : RBMCSA, 2006. v. 1. p. 1-4.

SILVA, R. C. L.; CARVALHO, R. J. D.; CALFA, B. A. Kinetcs and mass transfer of atrazine ozonization. Revista Ambiente \& Água., v.5, n. 2, p. 9-20, 2010.

SILVA, J. C. C. Desenvolvimento de Metodologia Analítica para determinação de Microcistina_Lr e agrotóxicos em águas superficiais,utilizando as técnicas de cromatografia líquida e cromatografia gasosa acopladas a espectometria de massa. 2010. 159p. Dissertação (Mestrado em Engenharia Ambiental). Universidade Federal de Ouro Preto, Ouro Preto, MG.

SNYDER, S. A.; WERT, E. C.; REXING, D. J.; ZEGERS, R. E.; DRURY, D. D. Ozone 0xidation of Endocrine Disruptors and Pharmaceuticals in Surface Water and Wastewater. Ozone: Science \& Engineering, v. 28, p. 445-460, 2006.

SOARES, A. F. S. Uso de agrotóxicos, contaminação de mananciais e análise da legislação pertinente: um estudo na região de Manhuaçu-MG. 2011. 294p. Tese (Doutorado em Saneamento, Meio Ambiente e Recursos Hídricos). Universidade Federal de Minas Gerais, Belo Horizonte, MG.

SOARES, A. F. S.; AZEVED0, B. A. D.; R0DRIGUES, N. U. A. Aplicação dos Algoritmos G0SS e GUS para Estimar a Contaminação das Águas de Mananciaias de Abastecimento Público. In: CONGRESSO BRASILEIR0 DE GESTÃo AMBIENTAL, 5., 2014, Belo Horizonte. Gestão Ambiental - Ações e Comportamento. Belo Horizonte: IBEAS, 2014. p. $1-5$.

SOLOMON, K. R.; CARR, J. A.; DU PREEZ, L. H.; GIESY, J. P.; KENDALL, R. J.; SMITH, E. E.; VAN DER KRAAK, G. J. Effects of atrazine on fish, amphibians, and aquatic reptiles: a critical review. Critical reviews in toxicology, v. 38, n. 9, p. 721-772, 2008.

SOUZA, V.; CARBO, L; DORES, E. F. G. C.; RIBEIR0, M. L.; VECCHIATO, A. B.; WEBER, O. L. S.; PINTO, A. A.; SPADOTTO, C. A.; CUNHA, M. L. F. Determinação de pesticidas em águas de poços tubulares em áreas de cultura de algodão na microrregião de primavera do leste, Mato Grosso. In: Congresso Brasileiro de Águas Subterrâneas, 13, 2004, Cuiabá. ABAS, 2004. p. 1-15.

THUY, P. T.; MOONS, K.; DIJK, J. C. V.; ANH, N. V.; BRUGGEN, B. V. der. To what extent are pesticides removed from surface water during coagulation-flocculation? Water and Environment Journal, v. 22, n. 3, p. 217-223, 2008.

USEPA. UNITED STATES ENVIRONMENTAL PROTECTION AGENCY. The Incorporation of Water Treatment Effects on Pesticide Removal and Transformations in Food Quality Protection Act (FQPA) Drinking Water Assessments. 2001

UNITED STATES ENVIRONMENTAL PROTECTION AGENCY. Disponível em: <https://safewater.zendesk.com/hc/en-us/sections/202366538-Atrazine〉. Acessado em 14 de março de 2018. (a)

UNITED STATES ENVIRONMENTAL PROTECTION AGENCY. Disponível em: 〈https://pubchem.ncbi.nlm.nih.gov/compound/2256\#section=Hazards-Summary . Acessado em 14 de março de 2018. (b) 
0corrência de Atrazina em águas no Brasil e remoção no tratamento da água: revisão sistemática

WHO - WORLD HEALTH ORGANIZATION . Guidelines for drinking water quality. Vol.1. 4rd ed. Versão eletrônica. 2017. Disponível em: <http://apps.who.int/iris/bitstream/handle/10665/254637/9789241549950eng.pdf;jsessionid=3FCA37069207D6A3E9B5117CCD3A1AE9?sequence=1>.Acessado em: 9 ago. 2017.

YIXIN, Y.; HONGBIN, C.; PAI, P.; HONGMIAO, B. Degradation and transformation of atrazine under catalyzedozonation process with Ti02as catalyst. Journal of Hazardous Materials, v. 279, p.444-451, 2014.

ZADAKA, D.; NIR, S.; RADIAN, A.; MISHAEL, Y. G. Atrazine removal from water by polycation-clay composites: Effect of dissolved organic matter and comparison to activated carbon. Water Research, v. 43, p. 677-683, 2009.

ZANINI, J. Estudo da remoção do herbicida atrazina por biofiltração em filtros lentos de areia e carvão ativado associada à ação microbiana. 2010. 98p. Dissertação (Mestrado em Engenharia Civil). Universidade Estadual Paulista, Ilha Solteira, SP.

ZHOU, Q.; WANG, W.; SHUANG, C.; WANG, M.; MA, Y.; LI, A. Reusable magnetic microspheres for efficient removal of atrazine in aqueous media. Chemical Engineering Journal, v. 253, p. 190-197, 2014. 